\title{
DIFFERENTIAL OPERATOR OF MEROMORPHIC $p$-VALENT FUNCTIONS
}

\author{
B. A. FRASIN
}

Abstract. A certain differential operator $I_{\lambda}^{m} f(z)$ is introduced for functions of the form $f(z)=\frac{1}{z^{p}}+\sum_{n=k}^{\infty} a_{n+p-1} z^{n+p-1}$ which are $p$-valent in the punctured unit disk $\mathscr{U}^{*}=\{z: z \in \mathbb{C}$ and $0<|z|<1\}$, where $p$ and $k$ are positive integers. The main object of this paper is to give an application of the operator $I_{\lambda}^{m} f(z)$ to the differential inequalities.

\section{Introduction and definitions}

Let $\sum_{p, k}$ denote the class of functions of the form :

$$
f(z)=\frac{1}{z^{p}}+\sum_{n=k}^{\infty} a_{n+p-1} z^{n+p-1}, \quad(p, k \in \mathbb{N}:=\{1,2,3, \ldots\}),
$$

which are $p$-valent in the punctured unit disk $\mathscr{U}^{*}=\{z: z \in \mathbb{C}$ and $0<|z|<1\}$. For a function $f$ in $\sum_{p, k}$, we define the following new differential operator:

$$
\begin{aligned}
& I^{0} f(z)=f(z), \\
& I_{\lambda}^{1} f(z)=(1-\lambda) f(z)+\lambda z f^{\prime}(z)+\frac{\lambda(p+1)}{z^{p}}, \quad \lambda \geq 0, \\
& I_{\lambda}^{2} f(z)=(1-\lambda) I_{\lambda}^{1} f(z)+\lambda z\left(I_{\lambda}^{1} f(z)\right)^{\prime}+\frac{\lambda(p+1)}{z^{p}},
\end{aligned}
$$

and for $m=1,2,3, \ldots$

$$
\begin{aligned}
I_{\lambda}^{m} f(z) & =(1-\lambda) I_{\lambda}^{m-1} f(z)+\lambda z\left(I_{\lambda}^{m-1} f(z)\right)^{\prime}+\frac{\lambda(p+1)}{z^{p}} \\
& =\frac{1}{z^{p}}+\sum_{n=k}^{\infty}[1+\lambda(p+n-2)]^{m} a_{n+p-1} z^{n+p-1}
\end{aligned}
$$

Note that for $\lambda=p=k=1$, we have the operator $I^{m} f(z)$ introduced and studied by Frasin and Darus [2]. 
It easily verified from (1.2) that

$$
\lambda z\left(I_{\lambda}^{m} f(z)\right)^{\prime}=I_{\lambda}^{m+1} f(z)-(1-\lambda) I_{\lambda}^{m} f(z)-\frac{\lambda(p+1)}{z^{p}} .
$$

From the identity (1.3), we readily have

$$
\lambda z\left(I_{\lambda}^{m-1} f(z)\right)^{\prime}=I_{\lambda}^{m} f(z)-(1-\lambda) I_{\lambda}^{m-1} f(z)-\frac{\lambda(p+1)}{z^{p}}
$$

and

$$
\lambda z\left(I_{\lambda}^{m+1} f(z)\right)^{\prime}=I_{\lambda}^{m+2} f(z)-(1-\lambda) I_{\lambda}^{m+1} f(z)-\frac{\lambda(p+1)}{z^{p}} .
$$

Very recently, Kamali [3] has obtained new properties of meromorphic $p$-valent functions defined by Ruscheweyh operator $D^{n+p-1} f(z)$ [6] (see also [7, 1, 4]).

The object of the present paper is to investigate some new properties of meromorphic $p$-valent functions by the above operator $I_{\lambda}^{m} f(z)$ given by (1.2).

Definition 1.1. Let $H$ be the set of complex valued functions $h(r, s, t): \mathbb{C}^{3} \rightarrow \mathbb{C}$ such that

$$
\begin{aligned}
& h(r, s, t) \text { is continuous in a domain } \mathbb{D} \subset \mathbb{C}^{3} ; \\
& (1,1,1) \in \mathbb{D} \text { and }|h(1,1,1)|<1 ; \\
& \left|h\left(e^{i \theta}, e^{i \theta}+\lambda \delta, \frac{\lambda^{2}(\delta+\beta)+3 \lambda \delta e^{i \theta}+e^{2 i \theta}}{e^{i \theta}+\lambda \delta}\right)\right| \geq 1
\end{aligned}
$$

whenever

$$
\left(e^{i \theta}, e^{i \theta}+\lambda \delta, \frac{\lambda^{2}(\delta+\beta)+3 \lambda \delta e^{i \theta}+e^{2 i \theta}}{e^{i \theta}+\lambda \delta}\right) \in \mathbb{D}
$$

with $\operatorname{Re} \beta \geq \delta(\delta-1)$ for real $\theta, \delta \geq 1$ and $\lambda>0$.

\section{Main result}

In order to prove our main result, we recall the following lemma due to Miller and Mocanu [5].

Lemma 2.1. Let $w(z)=a+w_{n} z^{n}+\cdots$, be analytic in $\mathscr{U}=\{z: z \in \mathbb{C}$ and $|z|<1\}$ with $w(z) \neq a$ and $n \geq 1$. If $z_{0}=r_{0} e^{i \theta}\left(0<r_{0}<1\right)$ and $\left|w\left(z_{0}\right)\right|=\max _{|z| \leq r_{0}}|w(z)|$. Then

$$
z w^{\prime}\left(z_{0}\right)=\delta w\left(z_{0}\right)
$$

and

$$
\operatorname{Re}\left\{1+\frac{z_{0} w^{\prime \prime}\left(z_{0}\right)}{w^{\prime}\left(z_{0}\right)}\right\} \geq \delta
$$

where $\delta$ is a real number and

$$
\delta \geq n \frac{\left|w\left(z_{0}\right)-a\right|^{2}}{\left|w\left(z_{0}\right)\right|^{2}-|a|^{2}} \geq n \frac{\left|w\left(z_{0}\right)\right|-|a|}{\left|w\left(z_{0}\right)\right|+|a|} .
$$


Theorem 2.2. Let $h(r, s, t) \in H$ and let $f(z) \in \sum_{p, k}$ satisfies

$$
\left(\frac{I_{\lambda}^{m} f(z)}{I_{\lambda}^{m-1} f(z)}, \frac{I_{\lambda}^{m+1} f(z)}{I_{\lambda}^{m} f(z)}, \frac{I_{\lambda}^{m+2} f(z)}{I_{\lambda}^{m+1} f(z)}\right) \in \mathbb{D} \subset \mathbb{C}^{3}
$$

and

$$
\left|h\left(\frac{I_{\lambda}^{m} f(z)}{I_{\lambda}^{m-1} f(z)}, \frac{I_{\lambda}^{m+1} f(z)}{I_{\lambda}^{m} f(z)}, \frac{I_{\lambda}^{m+2} f(z)}{I_{\lambda}^{m+1} f(z)}\right)\right|<1
$$

for all $z \in \mathscr{U}$ and for some $m \in \mathbb{N}$. Then we have

$$
\left|\frac{I_{\lambda}^{m} f(z)}{I_{\lambda}^{m-1} f(z)}\right|<1 \quad(z \in \mathscr{U} ; \lambda>0) .
$$

Proof. Let

$$
\frac{I_{\lambda}^{m} f(z)}{I_{\lambda}^{m-1} f(z)}=w(z) .
$$

Then it follows that $w(z)$ is either analytic or meromorphic in $\mathscr{U}, w(0)=1$ and $w(z) \neq 1$. Differentiating (2.5) logarithmically and multiply by $z$, we obtain

$$
\frac{z\left(I_{\lambda}^{m} f(z)\right)^{\prime}}{I_{\lambda}^{m} f(z)}-\frac{z\left(I_{\lambda}^{m-1} f(z)\right)^{\prime}}{I_{\lambda}^{m-1} f(z)}=\frac{z w^{\prime}(z)}{w(z)} .
$$

Using the identities (1.3) and (1.4), we have

$$
\frac{I_{\lambda}^{m+1} f(z)}{I_{\lambda}^{m} f(z)}=w(z)+\lambda \frac{z w^{\prime}(z)}{w(z)} .
$$

Differentiating (2.6) logarithmically and multiply by $z$, we have

$$
\begin{aligned}
\frac{z\left(I_{\lambda}^{m+1} f(z)\right)^{\prime}}{I_{\lambda}^{m+1} f(z)}-\frac{z\left(I_{\lambda}^{m} f(z)\right)^{\prime}}{I_{\lambda}^{m} f(z)} & =\frac{z\left[w(z)+\lambda \frac{z w^{\prime}(z)}{w(z)}\right]^{\prime}}{w(z)+\lambda \frac{z w^{\prime}(z)}{w(z)}} \\
& =\frac{z w^{\prime}(z)+\lambda\left[\frac{z w^{\prime}(z)}{w(z)}+\frac{z^{2} w^{\prime \prime}(z)}{w(z)}-\left(\frac{z w^{\prime}(z)}{w(z)}\right)^{2}\right]}{w(z)+\lambda \frac{z w^{\prime}(z)}{w(z)}} .
\end{aligned}
$$

Using the identities (1.3) and (1.5), it follows from (2.7) that

$$
\begin{aligned}
\frac{1}{\lambda} \frac{I_{\lambda}^{m+2} f(z)}{I_{\lambda}^{m+1} f(z)} & =\frac{1}{\lambda} \frac{I_{\lambda}^{m+1} f(z)}{I_{\lambda}^{m} f(z)}+\frac{z w^{\prime}(z)+\lambda\left[\frac{z w^{\prime}(z)}{w(z)}+\frac{z^{2} w^{\prime \prime}(z)}{w(z)}-\left(\frac{z w^{\prime}(z)}{w(z)}\right)^{2}\right]}{w(z)+\lambda \frac{z w^{\prime}(z)}{w(z)}} \\
& =\frac{1}{\lambda} w(z)+\frac{z w^{\prime}(z)}{w(z)}+\frac{z w^{\prime}(z)+\lambda\left[\frac{z w^{\prime}(z)}{w(z)}+\frac{z^{2} w^{\prime \prime}(z)}{w(z)}-\left(\frac{z w^{\prime}(z)}{w(z)}\right)^{2}\right]}{w(z)+\lambda \frac{z w^{\prime}(z)}{w(z)}}
\end{aligned}
$$


We claim that $|w(z)|<1$ for $z \in \mathscr{U}$. Otherwise there exists a point $z_{0} \in \mathscr{U}$ such that $\max _{|z| \leq r_{0}}|w(z)|=$ $\left|w\left(z_{0}\right)\right|=1$. Letting $w\left(z_{0}\right)=e^{i \theta}$ and using Lemma 2.1 with $a=1$ and $n=1$, we have

$$
\begin{aligned}
& \frac{I_{\lambda}^{m} f\left(z_{0}\right)}{I_{\lambda}^{m-1} f\left(z_{0}\right)}=e^{i \theta}, \\
& \frac{I_{\lambda}^{m+1} f\left(z_{0}\right)}{I_{\lambda}^{m} f\left(z_{0}\right)}=e^{i \theta}+\lambda \delta, \\
& \frac{I_{\lambda}^{m+2} f\left(z_{0}\right)}{I_{\lambda}^{m+1} f\left(z_{0}\right)}=\frac{\lambda^{2}(\delta+\beta)+3 \lambda \delta e^{i \theta}+e^{2 i \theta}}{e^{i \theta}+\lambda \delta},
\end{aligned}
$$

where

$$
\beta=\frac{z_{0}^{2} w^{\prime \prime}\left(z_{0}\right)}{w\left(z_{0}\right)} \quad \text { and } \quad \delta \geq 1 .
$$

Further, an application of (2.2) in Lemma 2.1 gives $\operatorname{Re} \beta \geq \delta(\delta-1)$.Since $h(r, s, t) \in H$, we have

$$
\begin{aligned}
& \left|h\left(\frac{I_{\lambda}^{m} f\left(z_{0}\right)}{I_{\lambda}^{m-1} f\left(z_{0}\right)}, \frac{I_{\lambda}^{m+1} f\left(z_{0}\right)}{I_{\lambda}^{m} f\left(z_{0}\right)}, \frac{I_{\lambda}^{m+2} f\left(z_{0}\right)}{I_{\lambda}^{m+1} f\left(z_{0}\right)}\right)\right| \\
= & \left|h\left(e^{i \theta}, e^{i \theta}+\lambda \delta, \frac{\lambda^{2}(\delta+\beta)+3 \lambda \delta e^{i \theta}+e^{2 i \theta}}{e^{i \theta}+\lambda \delta}\right)\right| \\
\geq & 1
\end{aligned}
$$

which contradicts the condition (2.4) of Theorem 2.2. Therefore, we conclude that

$$
\left|\frac{I_{\lambda}^{m} f(z)}{I_{\lambda}^{m-1} f(z)}\right|<1 \quad(z \in \mathscr{U}) .
$$

The proof is complete.

Letting $\lambda=1$ and $m=1$ in Theorem 2.2, we have

Corollary 2.3. Let $h(r, s, t) \in H$ and let $f(z) \in \sum_{p, k}$ satisfies

$$
\left(\begin{array}{l}
\frac{z^{p+1} f^{\prime}(z)+p+1}{z^{p} f(z)}, \frac{z^{p+2} f^{\prime \prime}(z)+z^{p+1} f^{\prime}(z)+1-p^{2}}{z^{p+1} f^{\prime}(z)+p+1}, \\
\frac{z^{p+3} f^{\prime \prime \prime}(z)+3 z^{p+2} f^{\prime \prime}(z)+z^{p+1} f^{\prime}(z)+1+p^{3}}{z^{p+2} f^{\prime \prime}(z)+z^{p+1} f^{\prime}(z)+1-p^{2}}
\end{array}\right) \in \mathbb{D} \subset \mathbb{C}^{3}
$$

and

$$
\left|h\left(\begin{array}{c}
\frac{z^{p+1} f^{\prime}(z)+p+1}{z^{p} f(z)}, \frac{z^{p+2} f^{\prime \prime}(z)+z^{p+1} f^{\prime}(z)+1-p^{2}}{z^{p+1} f^{\prime}(z)+p+1}, \\
\frac{z^{p+3} f^{\prime \prime \prime}(z)+3 z^{p+2} f^{\prime \prime}(z)+z^{p+1} f^{\prime}(z)+1+p^{3}}{z^{p+2} f^{\prime \prime}(z)+z^{p+1} f^{\prime}(z)+1-p^{2}}
\end{array}\right)\right|<1
$$

for all $z \in \mathscr{U}$. Then we have

$$
\left|\frac{z^{p+1} f^{\prime}(z)+p+1}{z^{p} f(z)}\right|<1 \quad(z \in \mathscr{U}) .
$$




\section{References}

[1] M. K. Aouf and H. M. Hossen, New criteria for meromorphic p-valent starlike functions, Tsukuba J. Math., 17(1993), 481-486.

[2] B. A. Frasin and M. Darus, On certain meromorphic functions with positive coefficients, South East Asian Bulletin of Math., 28(2004), 615-623.

[3] M. Kamali, On certain meromorphic p-valent starlike functions, J. Franklin Institute, 344(2007), 867-872.

[4] J. Liu and S. Owa, On certain meromorphic p-valent functions, Taiwanese J. Math., 2(1998), $107-110$.

[5] S. S. Miller and P. T. Mocanu, Second order differential inequalities in the complex plane, J. Math. Anal. Appl., 65 (1978), 289-305.

[6] S. Ruscheweyh, New criteria for univalent functions, Proc. Amer. Math. Soc., 49 (1975), 109-115.

[7] B. A. Uralegaddi and Somanatha, New criteria for meromorphic starlike univalent functions, Bull. Austral. Math. Soc., 43 (1991), 137-140.

Faculty of Science, Department of Mathematics, Al al-Bayt University, P.O. Box: 130095 Mafraq, Jordan.

E-mail: bafrasin@yahoo.com 